\title{
SEROTONIN SYNDROME INDUCED BY A SINGLE DOSE OF VENLAFAXINE AND MAGNESIUM VALPROATE
}

\author{
Zuan Zhan, Chun-shui Cao \& Liang Huang \\ Emergency Department, The First Affiliated Hospital of Nanchang University, Jiangxi, China
}

received: 27.10.2020;

revised: 15.1.2021;

accepted: 4.2.2021

\section{INTRODUCTION}

Serotonin syndrome (SS) is an adverse drug reaction resulting from therapeutic drug use, intentional selfpoisoning, or unintentional drug-drug interactions. It is especially common among patients who are prescribed with psychiatric drugs. The condition, if left untreated, can be potentially life-threatening. This case report outlines a patient with depression who developed SS after consuming a single normal dose of venlafaxine and magnesium valproate. To date, no similar cases have been reported in the literature to the best of our knowledge.

\section{CASE REPORT}

A 18-years old man about $70 \mathrm{~kg}$ with a history of depression presented to the emergency department in a manic state with psychotic features. According to his father, the patient consumed venlafaxine sustained-release tablets $(75 \mathrm{mg})$ and magnesium valproate sustained-release tablets $(0.25 \mathrm{~g})$ five hours ago. However, approximately two hours after ingesting the medication, he developed nausea and involuntary tremor of the lips. About 3 hours later, he became sweaty, uncontrollable, and delirious with systemic tremors. This was the first time he took these medications since he was diagnosed with depression two days ago at the psychosomatic medicine outpatient department. Prior to the diagnosis, he suffered from decreased interest and insomnia for two months. Apart from that, he had no other illnesses. There was also no exposure to drugs or toxic substances and he did not consume any alcohol.

On admission, the vital signs showed pulse rate of 155 beats/min, respiration rate of 26 breaths $/ \mathrm{min}$, blood pressure of $102 / 68 \mathrm{mmHg}$ and body temperature of $38.2^{\circ}$. The physical examination revealed that he was delirious and restless. Both pupils were dilated with a diameter of $4.5 \mathrm{~mm}$ and no light reflex. Nystagmus could be observed. Both of his lips and limbs were spasmodic. There were no rales in both lungs and no heart murmur. The abdomen was soft and non-tender on palpation. Hyperactive bowel sounds could be heard on auscultation. The bladder was full. There was resistance in the neck. The muscles in the limbs were tense and the tendons were overactive. Blood chemistry tests revealed elevated levels of ALT 55 U/L (9 50), AST 50 U/L (15 40), CK 7109 U/L $(50 \sim 310)$, CKMB 66 U/L (0 24), LDH $495 \mathrm{U} / \mathrm{L}$ (120 250), and lactic acid 3.0 $\mathrm{mmol} / \mathrm{L}(0.5 \sim 1.6)$. Bedside ECG was performed and it showed sinus tachycardia. CT Brain scan and full blood count were normal.

In view of the clinical manifestations that were highly consistent with SS, the family was informed to discontinue the use of venlafaxine and magnesium valproate. Although cyproheptadine was the preferred treatment for severe SS, it was not available in our hospital. Thus, midazolam was used in this patient to control the main symptoms. It was given intravenously at $0.1 \mathrm{mg} / \mathrm{kg} / \mathrm{h}$ via a syringe pump. He was also given a copious amount of fluids to promote drug excretion as well as sodium bicarbonate to alkalise the urine. An urinary catheter was inserted and nutritional support treatment was put in place. After about 72 hours, the patient regained calmness and lucidness. All abnormal symptoms disappeared. After 5 days, he was discharged from the hospital with a normal level of creatine kinase.

\section{DISCUSSION}

SS is a result of an excessive agonistic reaction in the serotonergic receptors of the central and peripheral nervous systems. The patients with SS commonly present with clinical signs such as autonomic nerve dysfunction, alteration in mental state, and neuromuscular dysfunction. To date, there are three sets of criteria used for the diagnosis of SS, namely Sternbach, Hunter, and Radomski (Werneke et al. 2016). Among them, the Hunter criteria is the most accurate diagnostic set available to diagnose SS (Dunkley et al. 2003).

Venlafaxine is a widely prescribed antidepressant medication. Its mechanism of action involves the blockage in the reuptake of serotonin and norepinephrine. As a result, venlafaxine may increase serotonin levels in the body and potentially cause SS. Furthermore, venlafaxine is metabolised to the active metabolite O-desmethylvenlafaxine by CYP2D6, and its level is, therefore, response-dependent on the variations in CYP2D6 metabolism. In the previously published case reports, SS has been described as a result of the usage of conventional doses of venlafaxine (Pan \& Shen 2003). However, more often than not, SS was caused by 
the overuse of venlafaxine and interaction with other drugs (Kolecki 1997) such as selective 5-HT uptake inhibitors and antiemetic drugs (Connor 2003).

On the other hand, magnesium valproate is a commonly prescribed antiepileptic drug. It is also being prescribed as adjuvant therapy for depression by some psychiatrists. Previous studies have shown an elevated concentration of serotonin in the central nervous system after the intake of valproate (Ahmad et al. 2005). Therefore, theoretically, the single use of this drug may also cause SS even though no cases have been reported thus far. Prakash et al. reported a case of SS that manifested a few days after the treatment of chronic migraine with a combination of amitriptyline and sodium valproate, thus suggesting a possible synergistic effect between amitriptyline and sodium valproate (Prakash et al. 2017).

In this case, it was the first time the patient was put on antidepressant. Unfortunately, he quickly developed autonomic nervous dysfunction, altered mental state, and neuromuscular dysfunction. He did not have any underlying diseases or similar episodes before. Furthermore, he did not have any other symptoms other than depression when taking the drug. There was also no ingestion of any other drugs or toxic substances. Upon admission, the differential diagnoses besides SS included central nervous system infection, malignant syndrome, and the patient's underlying mental illness. However, none of the remaining diagnoses could sufficiently explain the patient's condition. SS was the most likely diagnosis for the patient according to the Sternbach's and Hunter's criteria.

To date, there are no published studies on such an early progression to SS after a single dose of venlafaxine and magnesium valproate. Since both drugs were capable of increasing the serotonin levels in the body and they were ingested at the same time, it became difficult to determine which drug resulted in the adverse drug reaction. Although a small dose of venlafaxine ( $37.5 \mathrm{mg} / \mathrm{d}$ ) had been reported by (Pan \& Shen 2003 ) to cause SS in a previous study, the time of symptom presentation was not as early and the symptoms were less severe than the patient in this case report. Furthermore, in the case report by (Pan \& Shen 2003), the patient was treated with imipramine for two weeks before receiving venlafaxine. Thus, it was possible that a pharmacodynamic interaction might have happened between the two medications. As for magnesium valproate, there were also no previous reports of SS being caused by the drug alone. Nevertheless, a previous study has shown that the combination of valproate and venlafaxine can result in an elevated level of O-desmethylvenlafaxine, the active metabolite of venlafaxine (Unterecker et al. 2014). In view of this, the possibility of a synergistic effect between the two drugs in this study was high. Due to the underlying genetic polymorphism, about $1 \%$ of the Chinese population do not possess the CYP2D6 enzymatic activity, thus affecting the metabolism of venlafaxine (Wang et al. 1993). Theoretically, this could lead to SS but the possibility was small for this patient because he was only prescribed with a small dose of venlafaxine. Upon resolution of the symptoms, he was recommended to restart the treatment with a smaller dose of venlafaxine. Unfortunately, the patient was not keen as he was afraid of a recurrence of SS.

\section{CONCLUSION}

In summary, this case serves as an important reminder to healthcare practitioners, especially psychiatrists that combination therapy of venlafaxine and valproate may pose as a risk of developing SS for patients who have never been treated with any antidepressant. For these patients, single-drug therapy would be safer. Additionally, healthcare practitioners must be aware of the presentation of SS to avoid misdiagnosis. It is vital for them to pay attention to the rapid progress of the disease in the early stage so that timely and effective treatment strategies can be undertaken to prevent unwanted morbidity and mortality.

\section{Acknowledgments:}

We would like to thank Emergency Department, The First Affiliated Hospital of Nanchang University for their continuous support. Thanks to the patient's mother for allowing us to share the details.

\section{Conflict of interest: None to declare.}

\section{Contribution of individual authors:}

Zuan Zhan: design, revisioning the manuscript, comments of the draft paper, approval of the final version.

Chun-shui Cao: revisioning the manuscript, comments of the draft paper.

Liang Huang: revisioning the manuscript, comments of the draft paper.

\section{References}

1. Ahmad S, Fowler LJ \& Whitton PS: Effects of combined lamotrigine and valproate on basal and stimulated extracellular amino acids and monoamines in the hippocampus of freely moving rats. Naunyn Schmiedebergs Arch Pharmacol 2005; 371:1-8

2. Connor H: Serotonin syndrome after single doses of coamoxiclav during treatment with venlafaxine. $J R$ Soc Med 2003; 96:233-4

3. Dunkley EJ, Isbister GK, Sibbritt D, Dawson AH \& Whyte IM: The Hunter serotonin toxicity criteria: simple and accurate diagnostic decision rules for serotonin toxicity. QJM 2003; 96:635-42

4. Kolecki P: Isolated venlafaxine-induced serotonin syndrome. J Emerg Med 1997; 15:491-3

5. Pan JJ \& Shen WW: Serotonin syndrome induced by lowdose venlafaxine. Ann Pharmacother 2003; 37:209-11

6. Prakash S, Adroja B \& Parekh H: Serotonin syndrome in patients with headache disorders. BMJ Case Rep 2017 
7. Unterecker S, Reif A, Hempel S, Proft F, Riederer P, Deckert $J$, et al.: Interaction of valproic acid and the antidepressant drugs doxepin and venlafaxine: analysis of therapeutic drug monitoring data under naturalistic conditions. Int Clin Psychopharmacol 2014; 29:206-11

8. Wang SL, Huang JD, Lai MD, Liu BH \& Lai ML: Molecular basis of genetic variation in debrisoquin hydroxylation in Chinese subjects: polymorphism in RFLP and DNA sequence of CYP2D6. Clin Pharmacol Ther 1993; 53:410-8

9. Werneke U, Jamshidi F, Taylor DM \& Ott M: Conundrums in neurology: diagnosing serotonin syndrome - a meta-analysis of cases. BMC Neurol 2016; 16:1-9

Correspondence:

Zuan Zhan, MD

Emergency Department, The First Affiliated Hospital of Nanchang University

Jiangxi, China

E-mail: 51173277@qq.com 